\title{
Successful management of traumatic pancreatic transection using ERCP-guided pancreatic stenting
}

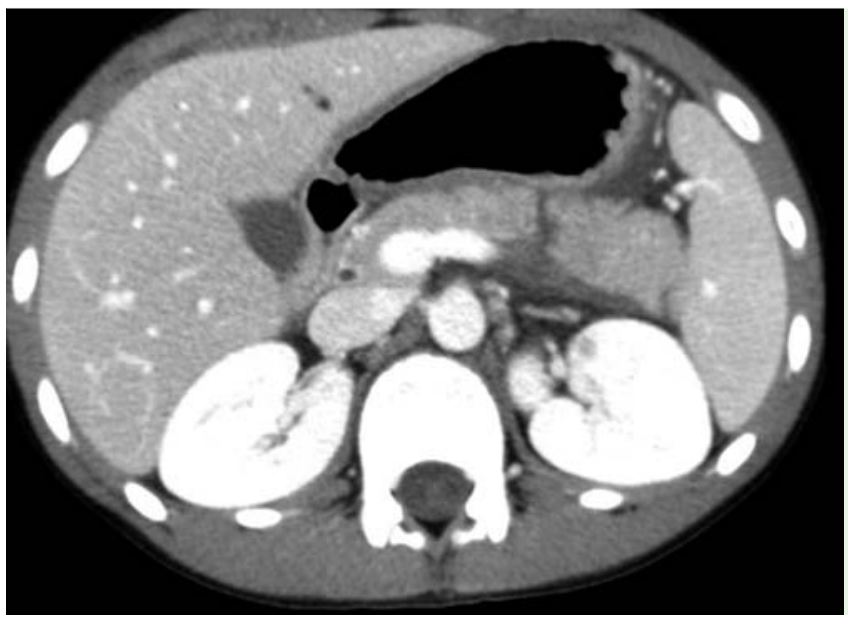

Fig. 1 Abdominal computed tomography (CT) scan (transverse view) of an 11-year-old boy after a cycling accident, showing complete pancreatic transection (type III).

An 11-year-old boy was admitted to the emergency department with severe abdominal pain and vomiting after falling off his bicycle. A round hematoma was noted in the left hypochondrium consistent with bicycle handlebar trauma. Blood analysis showed mild leucocytosis and marked elevation of lipase (2507U/L) and amylase (1331 U/L). Abdominal ultrasound excluded spleen injury, and thoracic X-ray was unremarkable.

Abdominal computed tomography (CT) scan showed a total transection of the pancreas, including the pancreatic duct, in the distal body, without liver, splenic, or vascular lesions ( $\bullet$ Fig. 1 and $\bullet$ Fig. 2). Endoscopic retrograde cholangiopancreatography (ERCP) was performed. Pancreatography revealed contrast extravasation from the main pancreatic duct in the distal body ( $\bullet$ Fig. 3, $\bullet$ Video 1 ). Cannulation of the caudal portion of the duct was not possible; therefore, a 5-Fr, 12-cm length, plastic monopigtail stent was placed with the distal extremity in the fistula.

The patient recovered well and was discharged after 20 days without pain, and

\section{Video 1}

Pancreatogram showing contrast extravasation and placement of the stent. with almost complete normalization of blood analysis.

After 1 month, ultrasound showed no signs of pancreatic injury. An ERCP was performed again, showing a well-positioned pancreatic stent that was subsequently removed using a forceps. Pancreatography showed a mild stenosis of the main duct associated with a cystic lesion ( $\bullet$ Fig. 4). The asymptomatic patient was discharged on the following day.

Isolated pancreatic transection with duct injury because of blunt abdominal trauma is very rare. Its management can be surgical, usually with distal pancreatectomy, or nonsurgical. Previous studies have shown successful results with both approaches, with more rapid resolution in the surgical group [1], but ERCP was not used for therapeutic purposes. In fact, only a few case reports have assessed the efficacy of this technique $[2,3]$. While clinically unstable patients need a more aggressive management, in those with clinical stability, ERCP-guided pancreatic stenting to ensure effective drainage seems to be a very successful approach.

Endoscopy_UCTN_Code_CCL_1AZ_2AI

Competing interests: None

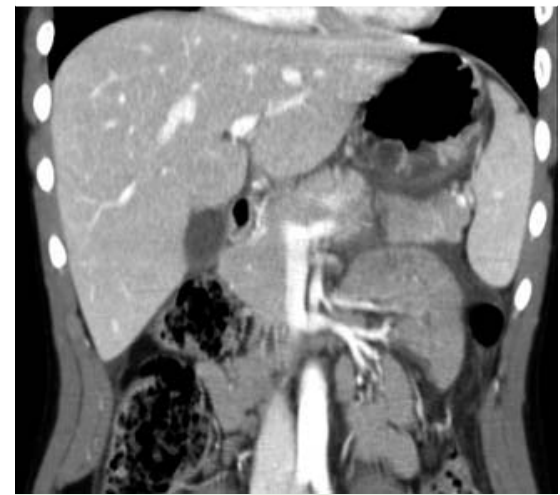

Fig. 2 Abdominal CT scan (coronal view) showing complete pancreatic transection (type III).

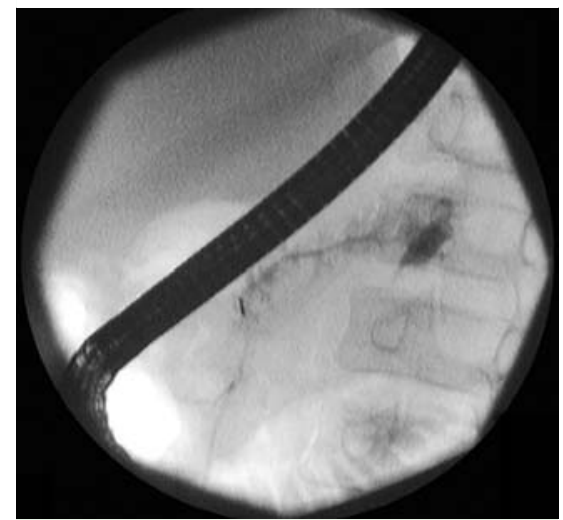

Fig. 3 Fluoroscopic images obtained with endoscopic retrograde cholangiopancreatography (ERCP) showing contrast extravasation from the main pancreatic duct.

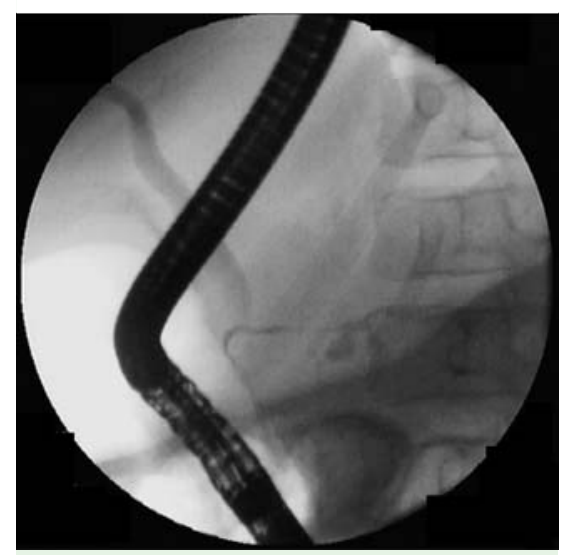

Fig. 4 Follow-up pancreatogram after 1 month showing mild pancreatic duct stenosis with associated cystic lesion. 
João Santos-Antunes ${ }^{1,2}$, Pedro Pereira', Eduardo Rodrigues-Pinto ${ }^{1}$, Armando Ribeiro' ${ }^{1}$ Carlos Mariz ${ }^{3}$, Guilherme Macedo ${ }^{1}$

${ }^{1}$ Department of Gastroenterology, Faculty of Medicine, Hospital de São João, Porto, Portugal

2 Department of Biochemistry (U38-FCT), Faculty of Medicine, University of Porto, Porto, Portugal

${ }^{3}$ Paediatric Surgery Department, Hospital de São João, Porto, Portugal

\section{References}

1 Iqbal CW, St Peter SD, Tsao Ket al.; Pancreatic Trauma in Children (PATCH) Study Group. Operative vs nonoperative management for blunt pancreatic transection in children: multi-institutional outcomes. J Am Coll Surg 2014; 218: $157-162$

2 Hiremath B, Hegde $N$. Non-operative management of a grade IV pancreatic injury. BMJ Case Rep 2014. DOI: 10.1136/bcr-2014203805

3 Magrill D, Paterson I. Traumatic transection of the pancreatic duct. BMJ Case Rep 2009. DOI: 10.1136/bcr.06.2008.0005
Bibliography

Dol http://dx.doi.org/ 10.1055/s-0034-1390861 Endoscopy 2015; 47: E112-E113 (C) Georg Thieme Verlag KG Stuttgart · New York ISSN 0013-726X

\section{Corresponding author} João Santos-Antunes, MD

Department of Gastroenterology Faculty of Medicine

Centro Hospitalar São João

Alameda Prof. Hernani Monteiro 420-319 Porto

Portugal

Fax: +351-22-5513601

joao.claudio.antunes@gmail.com 